\title{
Assessing Pulmonary Perfusion in Emphysema
}

\section{Automated Quantification of Perfused Blood Volume in Dual-Energy CTPA}

\author{
Felix G. Meinel, MD, * Anita Graef, * Sven F. Thieme, MD, * Fabian Bamberg, MD, MPH, * \\ Florian Schwarz, MD, * Wieland H. Sommer, MD, * Andreas D. Helck, MD, * Claus Neurohr, MD, $\dagger$ \\ Maximilian F. Reiser, MD,* and Thorsten R.C. Johnson, MD*
}

\begin{abstract}
Objectives: The objective of this study was to determine whether automated quantification of lung perfused blood volume (PBV) in dual-energy computed tomographic pulmonary angiography (DE-CTPA) can be used to assess the severity and regional distribution of pulmonary hypoperfusion in emphysema. Materials and Methods: We retrospectively analyzed 40 consecutive patients (mean age, 67 [13] years) with pulmonary emphysema, who have no cardiopulmonary comorbidities, and a DE-CTPA negative for pulmonary embolism. Automated quantification of global and regional pulmonary PBV was performed using the syngo Dual Energy application (Siemens Healthcare). Similarly, the global and regional degrees of parenchymal hypodensity were assessed automatically as the percentage of voxels with a computed tomographic density less than -900 Hounsfield unit. Emphysema severity was rated visually, and pulmonary function tests were obtained by chart review, if available.
\end{abstract}

Results: Global PBV generated by automated quantification of pulmonary PBV in the DE-CTPA data sets showed a moderately strong but highly significant negative correlation with residual volume in percentage of the predicted residual volume $(r=-0.62 ; P=0.002 ; n=23)$ and a positive correlation with forced expiratory volume in 1 second in percentage of the predicted forced expiratory volume in 1 second $(r=0.67 ; P<0.001 ; n=23)$. Global PBV values strongly correlated with diffusing lung capacity for carbon monoxide $(r=0.80$; $P<0.001 ; n=15)$. Pulmonary PBV values decreased with visual emphysema severity $(r=-0.46, P=0.003, n=40)$. Moderate negative correlations were found between global PBV values and parenchymal hypodensity both in a per-patient $(r=-0.63 ; P<0.001 ; n=40)$ and per-region analyses $(r=-0.62$; $P<0.001 ; n=40)$.

Conclusions: Dual-energy computed tomographic pulmonary angiography allows simultaneous assessment of lung morphology, parenchymal density, and pulmonary PBV. In patients with pulmonary emphysema, automated quantification of pulmonary PBV in DE-CTPA can be used for a quick, reader-independent estimation of global and regional pulmonary perfusion, which correlates with several lung function parameters.

Key Words: pulmonary emphysema, severity, distribution, pulmonary perfusion, dual-energy CT

(Invest Radiol 2013;48: 79-85)

$\mathrm{C}$ hronic obstructive pulmonary disease (COPD) is one of the leading causes of morbidity and mortality worldwide. ${ }^{1,2}$ It is characterized by airflow limitation and lung inflammation resulting

Received for publication July 25, 2012; and accepted for publication, after revision, September 19, 2012

From the Departments of *Clinical Radiology, and $†$ Internal Medicine, Ludwig Maximilians-University, Munich, Germany.

Felix G. Meinel and Anita Graef contributed equally to this work

Conflicts of interest and sources of funding: none declared.

Reprints: Felix G. Meinel, MD, Department of Clinical Radiology, Ludwig Maximilians-University, Großhadern Campus, Marchioninistr. 1581377 Munich, Germany. E-mail: felix.meinel@med.uni-muenchen.de.

Copyright (C) 2013 by Lippincott Williams \& Wilkins

ISSN: 0020-9996/13/4802-0079 in a progressive deterioration in lung function. Emphysema is a common component of COPD, in which airway obstruction, inflammation, and aberrant activity of proteolytic enzymes cause irreversible destruction of the alveolar walls and enlargement of distal airspaces. $^{3}$

Currently, screening for and early diagnosis of COPD and emphysema largely rely on spirometric lung function tests. ${ }^{4}$ Spirometry, however, strongly depends on patients' cooperation and is unable to localize emphysematous changes within the lung. Because pulmonary emphysema involves a complex pathophysiology of parenchymal destruction and hypoxic vasoconstriction, the correlation between areas of parenchymal destruction and areas of hypoperfusion is known to be strong but imperfect. ${ }^{5,6}$ This reflects the complex pathophysiology of pulmonary emphysema in which airflow obstruction, parenchymal destruction, and hypoxic vasoconstriction all contribute to impaired gas exchange.

Assessing the regional distribution of pulmonary emphysema and integrating the complementary information of lung morphology and lung perfusion are particularly relevant in the assessment of patients with emphysema who are considered for lung volume reduction surgery (LVRS) or endobronchial interventions. ${ }^{7,8}$ A study in 25 patients showed a superior prediction of postoperative outcome after LVRS for coregistered perfusion single-photon emission computed tomography (SPECT) and computed tomography (CT) as compared with planar scintigraphy, stand-alone SPECT, or qualitative assessment of stand-alone CT. ${ }^{9}$ Data from more than 1000 patients undergoing LVRS within the National Emphysema Treatment Trial showed that LVRS reduces mortality in patients with upper lobe-predominant emphysema only if there is low, rather than high, perfusion to the upper lung on scintigraphy. ${ }^{10}$ Therefore, imaging of pulmonary emphysema should ideally assess the regional distribution of both parenchymal destruction and pulmonary perfusion simultaneously.

Dual-energy computed tomographic pulmonary angiography (DE-CTPA) can be used to generate iodine distribution maps of pulmonary parenchyma, ${ }^{11-15}$ which correspond well with pulmonary perfusion on scintigraphy ${ }^{16}$ and SPECT ${ }^{17}$ images. Dual-energy CT thus simultaneously provides high-resolution morphological images of pulmonary parenchyma and functional information on pulmonary perfusion. A small number of initial studies have applied dual-energy $\mathrm{CT}$ to the quantification of pulmonary PBV in emphysema. ${ }^{6,18,19}$ These studies have confirmed a good but imperfect correlation between areas of hypodensity on virtual noncontrast scans and areas of PBV defects on iodine distribution maps. , $^{6}$

A recently developed software allows automated quantification of the iodine concentration in pulmonary parenchyma, potentially providing a quick, reader-independent tool for the assessment of global and regional pulmonary PBV. ${ }^{20}$ The purpose of this study was to assess whether automated quantification of pulmonary PBV in DECTPA can be used to quantify global and regional pulmonary perfusion in pulmonary emphysema and to correlate DE-CTPA-derived PBV values with visual, densitometric, and functional assessments of pulmonary emphysema. 


\section{MATERIALS AND METHODS}

\section{Computed Tomographic Image Acquisition}

All DE-CTPAs were performed on a dual-source CT scanner (SOMATOM Definition Flash; Siemens Medical, Forchheim, Germany). An $85 \mathrm{~mL}$ of contrast material (iopromide, Ultravist 370; Bayer Schering Pharma, Berlin, Germany) was administered via an antecubitalvein at a flow rate of $5 \mathrm{~mL} / \mathrm{s}$, followed by $50 \mathrm{~mL}$ of saline, which was injected at the same flow rate. Scans were started using a bolus-tracking technique with a threshold of 100 Hounsfield unit (HU) in the pulmonary trunk and an additional delay of 7 seconds. To reduce streak artifacts caused by dense contrast material in the superior vena cava, scans were performed in caudocranial direction. A combination of a tin-filtered (Sn) 140-kilovolt (peak) (kV[p]) and a $100-\mathrm{kV}(\mathrm{p})$ spectrum was used. Collimation was set to $32 \times 0.6 \mathrm{~mm}$. Pitch was 0.5 at a rotation time of $0.28 \mathrm{~s} /$ rot. Mean (SD) computed tomography dose index and dose length product were 10.0 (2.5) $\mathrm{mGy}$ and $315(85) \mathrm{mGy} \cdot \mathrm{cm}$, corresponding to an effective radiation dose of $4.6 \mathrm{mSv}$ (using a standard conversion factor for chest $\mathrm{CT}$ of $\left.0.0145 \mathrm{mSv} / \mathrm{mGy} \times \mathrm{cm}^{21}\right)$.

\section{Computed Tomographic Image Reconstruction}

Computed tomographic pulmonary angiographic (CTPA) images were reconstructed in axial orientation using a specific medium soft convolution kernel optimized for dual-energy (DE) images (D30) at a $1.5-\mathrm{mm}$ slice thickness with a $1.0-\mathrm{mm}$ increment. The tin-filtered $140-\mathrm{kV}(\mathrm{p})$ spectrum of the second-generation dual-source scanner in this study allows for using $100 \mathrm{kV}(\mathrm{p})$ as the second spectrum instead of the $80-\mathrm{kV}(\mathrm{p})$ spectrum used in most published studies because the filter decreases spectral overlap between both tubes. If this spectral combination of $\mathrm{Sn} 140 \mathrm{kV}(\mathrm{p})$ and $100 \mathrm{kV}(\mathrm{p})$ is used, an average image with equal contributions from both spectra best corresponds to a singlespectrum scan at $120 \mathrm{kV}(\mathrm{p})$. Therefore, average images were generated with equal contributions from the $\mathrm{Sn} 140-\mathrm{kV}(\mathrm{p})$ and the $100-\mathrm{kV}(\mathrm{p})$ data set. Dual-energy CT imaging allows for material differentiation based on the different absorption characteristics of different types of tissue. Iodine is known to produce higher attenuation at lower tube voltage settings. ${ }^{22}$ Thus, the spectral information obtained at different voltage settings allows for a 3-material decomposition differentiating soft tissue, air, and iodine. This algorithm assigns a ratio of air and soft tissue to each voxel and uses CT attenuation values at both energies to derive the additional iodine content. ${ }^{23}$ Virtual noncontrast images are generated by subtracting enhancement due to iodine from the average images. Color-coded iodine distribution maps were generated by specific, Food and Drug Administration-approved DE postprocessing software ("lung PBV" in syngo Dual Energy; Siemens Healthcare) on a dedicated postprocessing workstation (syngo multimodality workplace, Somaris Version CT2008G; Siemens Healthcare). Iodine distribution PBV maps were superimposed onto CTPA images at a slice thickness of $3 \mathrm{~mm}$ with a $1-\mathrm{mm}$ increment in axial, sagittal, and coronal orientations.

\section{Image Quality Control}

To ensure valid assessment of pulmonary PBV, we excluded all examinations with substantial artifacts, inadequate enhancement of the pulmonary vasculature, and/or inadequate timing. For this purpose, all images considered for the study were reviewed. The degree of artifacts in iodine distribution maps (from cardiac motion, breathing, or concentrated contrast material) was rated on a 4-point scale $(0$, no artifacts; 1 , mild artifacts; 2 , moderate artifacts; and 3 , severe artifacts). All studies with severe artifacts were excluded from the study. In addition, we measured enhancement in the pulmonary trunk and the ascending aorta. Because lung PBV values are quantified by measuring the enhancement of the pulmonary parenchyma relative to the enhancement of the pulmonary trunk, PBV values may become unreliable with inadequate enhancement of the pulmonary vasculature and inadequate timing of the examination relative to the contrast bolus. Therefore, all examinations with an enhancement of the pulmonary trunk of less than $200 \mathrm{HU}$ or less than the enhancement of the ascending aorta were excluded. Because this study was performed on a second-generation dual-source scanner with an extended field of view of $32 \mathrm{~cm}$ for dual-energy applications, the dual-energy analysis fully covered the pulmonary parenchyma in all patients.

\section{Patient Selection}

We defined the following inclusion criteria:

1. DE-CTPA performed for clinical indication of suspected acute pulmonary embolism

2. DE-CTPA negative for pulmonary embolism

3. Known pulmonary emphysema and/or pulmonary emphysema detected on DE-CTPA

4. Informed consent provided

We defined the following exclusion criteria:

1. DE-CTPA with severe artifacts and inadequate vascular enhancement or timing

2. Any significant cardiopulmonary comorbidities (including signs of congestive heart failure, pneumonia, lung masses, significant pleural effusions or atelectasis, pneumothorax, and others)

All DE-CTPAs performed at our institution between May 2009 and November 2011 were assessed for the presence of pulmonary embolism, pulmonary emphysema, and any cardiopulmonary comorbidities that might influence pulmonary PBV. We included all 40 patients with known or newly detected pulmonary emphysema, who have no cardiopulmonary comorbidities, and DE-CTPA negative for pulmonary embolism.

\section{Ethical Approval and Informed Consent}

Written informed consent for the CTPA examination was obtained from all patients. Ethical approval and individual informed consent for the retrospective data analysis were waived by the responsible ethics committee.

\section{Computed Tomographic Data Analysis}

One board-certified radiologist with more than 10 years of experience in thoracic imaging (T.R.C.J.) and 1 radiology resident (F.G.M.) reviewed the reconstructed CTPA images in consensus, blinded to the patients' clinical data. Images were analyzed in axial, sagittal, and coronal orientations on a postprocessing workstation (syngo multimodality workplace using 3-dimensional mode).

\section{Visual Rating of Emphysema Severity}

Emphysema severity was rated on virtual noncontrast images using a 5-point scale. The emphysema score was assigned on the basis of emphysematous area involvement: 1 , less than $5 \%$ (minimal); $2,5 \%$ to $25 \%$ (mild); $3,26 \%$ to $50 \%$ (moderate); $4,51 \%$ to $75 \%$ (severe); and 5 , more than $75 \%$ (very severe). ${ }^{24}$ The predominant morphology of emphysema was classified as centrilobular, panacinar, paraseptal, or mixed/equivocal. Bullous emphysema was not considered a distinct subtype because bullous changes commonly occur in all subtypes of advanced emphysema.

\section{Automated Quantification of Pulmonary PBV}

Automated quantification of pulmonary PBV was performed using the DE lung PBV application of the syngo Dual Energy software (version VE32B; Siemens Healthcare). This analysis quantifies pulmonary PBV by measuring the enhancement of the pulmonary parenchyma in relation to the enhancement of a reference vessel. The reference vessel was defined by placing a standardized region of 
interest sized $0.5 \mathrm{~cm}^{2}$ in the pulmonary trunk on axial images. The software calculates pulmonary PBV as (mean enhancement of the pulmonary parenchyma in HU/mean enhancement of the pulmonary trunk in HU)/calibration factor 0.15 . For example, for a vascular enhancement of $400 \mathrm{HU}$, a mean enhancement of the pulmonary parenchyma of $400 \mathrm{HU} \times 0.15=60 \mathrm{HU}$ would represent a PBV of $100 \%$. Lung isolation, lung partitioning (in the upper, middle, and lower zones), and enhancement analysis are performed automatically. The software then displays PBV for the entire pulmonary parenchyma, the right and left lungs separately, and the lower, middle, and upper zones of each lung separately.

\section{Analysis of Parenchymal Density}

The identical software (DE lung PBV application of the syngo Dual Energy software, version VE32B; Siemens Healthcare) was used to quantify the percentage of voxels with attenuation values lower than $-900 \mathrm{HU}$ in the virtual noncontrast images for the entire pulmonary parenchyma, the right and left lungs separately, and the lower, middle, and upper zones of each lung separately. This cutoff was chosen based on the literature such that healthy lungs would have $<5 \%$ of voxels of this low density, thus making it highly specific for emphysematous parenchyma. ${ }^{25}$ Zones were automatically defined by the software and were identical to the zones used for PBV quantification.

\section{Analysis of Pulmonary Function Tests}

Because pulmonary emphysema can progress over time, we only included pulmonary function tests (PFTs) performed within 6 months of the CTPA examination. For 23 of the 40 patients, PFTs performed within 6 months of the DE-CTPA examination were available from the patients' charts. The forced expiratory volume in 1 second $\left(\mathrm{FEV}_{1}\right)$ in percentage of the predicted $\mathrm{FEV}_{1}$ and the residual volume (RV) in percentage of the predicted RV, as determined by body plethysmography, were recorded and analyzed. The diffusing capacity for carbon monoxide (DLCO) had been assessed in 15 of these patients. If available, DLCO was recorded and included in the analysis.

\section{Statistical Analysis}

Statistical analysis was performed using Microsoft Excel for Mac 2001 (version 14.1.3) and IBM SPSS statistics for Mac (version 20.0.0.1). Global PBV values were correlated with visual rating scores of emphysema severity, global parenchymal hypodensity (the percentage of voxels with attenuation values lower than $-900 \mathrm{HU}$ ), and parameters of pulmonary function. Regional PBV values were correlated with regional parenchymal hypodensity. The Spearman correlation coefficient $r$ was used for all correlation analyses. Values of $r$ from 0 to 0.29 , from 0.30 to 0.69 , and greater than 0.70 were considered weak, moderate, and strong correlations. ${ }^{26}$ A 2-tailed $P<$ 0.05 was considered statistically significant. Multivariate regression analysis was performed to adjust for the influence of age and sex. For the region-based analysis, we also adjusted for the global pulmonary PBV values.

\section{RESULTS}

\section{Patient Characteristics}

Mean (SD) age was 67 (13) years with a range of 39 to 90 years (Table 1). Of the 40 patients, 17 (43\%) were women. Emphysema was classified as predominantly centrilobular in 28 patients (70\%), panacinar in 8 patients $(20 \%)$, paraseptal in 1 patient $(3 \%)$, and equivocal in 3 patients $(8 \%)$. Computed tomographic-morphologic evidence of emphysema severity was rated as minimal in 8 patients $(20 \%)$, mild in 12 patients $(30 \%)$, moderate in 9 patients $(23 \%)$, severe in 7 patients $(18 \%)$, and very severe in 4 patients $(10 \%)$.

\section{Correlation of Global Pulmonary PBV Values With Age}

After the adjustment for the visual severity of emphysema, there was no significant correlation between PBV values and age $(\beta=0.01 ; P=0.96)$.

\section{Correlation of the Global Pulmonary PBV Values With Visual Rating and Attenuation-Based Emphysema Quantification}

A moderate but significant negative correlation was found between visual rating scores of emphysema severity and the global pulmonary PBV values $(r=-0.46 ; P=0.003$; Fig. 1A), which was confirmed as an independent correlation in multivariate regression analysis $(\beta=-0.35 ; P=0.02)$. This correlation was not significant for mild to moderate emphysema (severity scores, 1-3). Similarly, there was a negative correlation between the global PBV values and the percentage of voxels within the pulmonary parenchyma with a CT density less than $-900 \mathrm{HU}$ on bivariate correlation $(r=-0.63 ; P<$ 0.001 ; Fig. 1B) and multivariate regression $(\beta=-0.54 ; P<0.001)$.

\section{Region-Based Analysis: Correlation of the Regional PBV Values and Parenchymal Density}

Next, we correlated the regional PBV values and parenchymal hypodensity for the upper, middle, and lower lung zones of each lung as defined by automatic lung partitioning. In this per-region analysis, a moderate correlation was observed between the regional PBV values and regional parenchymal hypodensity quantified as the regional percentage of voxels with a CT density lower than $-900 \mathrm{HU}$ $(r=-0.62 ; P<0.001$; Fig. 1C). On multivariate regression analysis, this correlation was independent of age, sex, and global PBV values $(\beta=-0.43 ; P<0.001)$.

\section{Correlation of the Global Pulmonary PBV Values With PFTs}

Global PBV generated by automated quantification in the DECTPA data sets showed a moderate but highly significant negative

TABLE 1. Patient Characteristics

\begin{tabular}{|c|c|}
\hline No. patients & 40 \\
\hline Indication for CTPA & Suspected pulmonary embolism \\
\hline Result of CTPA & No pulmonary embolism \\
\hline \multicolumn{2}{|l|}{ Emphysema } \\
\hline $\begin{array}{l}\text { Known and confirmed on index } \\
\text { CTPA, n (\%) }\end{array}$ & $17(43 \%)$ \\
\hline $\begin{array}{l}\text { Incidentally detected on index } \\
\text { CTPA, n (\%) }\end{array}$ & $23(57 \%)$ \\
\hline Cardiopulmonary comorbidities & None \\
\hline Age, mean (SD) [range], y & 67 (13) [39-90] y \\
\hline Women, n $(\%)$ & $17(43 \%)$ \\
\hline \multicolumn{2}{|l|}{ Emphysema type } \\
\hline centrilobular, n (\%) & $28(70 \%)$ \\
\hline panacinar, n (\%) & $8(20 \%)$ \\
\hline paraseptal, n (\%) & $1(3 \%)$ \\
\hline equivocal, n (\%) & $3(8 \%)$ \\
\hline \multicolumn{2}{|l|}{ Emphysema severity } \\
\hline minimal, n (\%) & $8(20 \%)$ \\
\hline mild, n (\%) & $12(30 \%)$ \\
\hline moderate, n (\%) & $9(23 \%)$ \\
\hline severe, $\mathrm{n}(\%)$ & $7(18 \%)$ \\
\hline very severe, n (\%) & $4(10 \%)$ \\
\hline
\end{tabular}




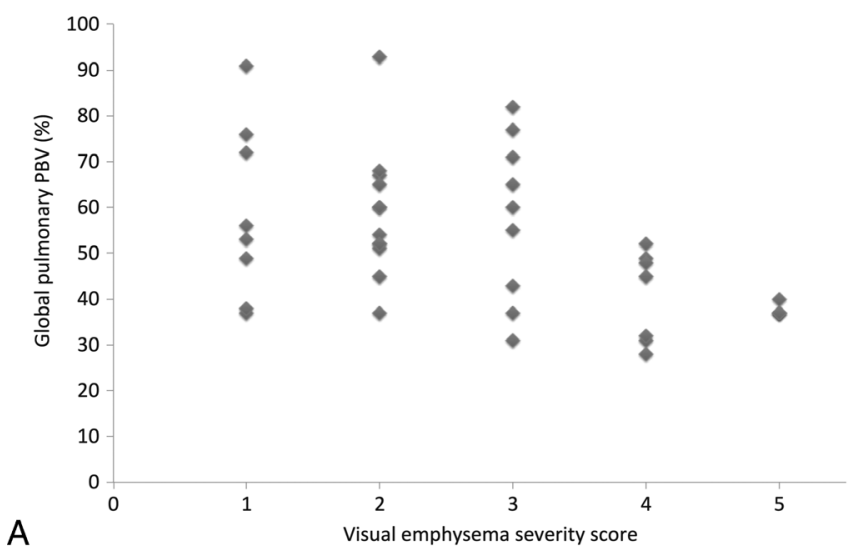

correlation $(r=-0.62 ; P=0.002)$ with $\mathrm{RV}$ in percentage of the predicted RV (Fig. 2A). This correlation was independent of confounding by age and sex, as confirmed by the multivariate regression analysis (standardized correlation coefficient $\beta=-0.33, P=0.006$ ). Similarly, a positive correlation was found between the global $\mathrm{PBV}$ values and $\mathrm{FEV}_{1}$ in percentage of the predicted $\mathrm{FEV}_{1}(\mathrm{r}=0.67$;
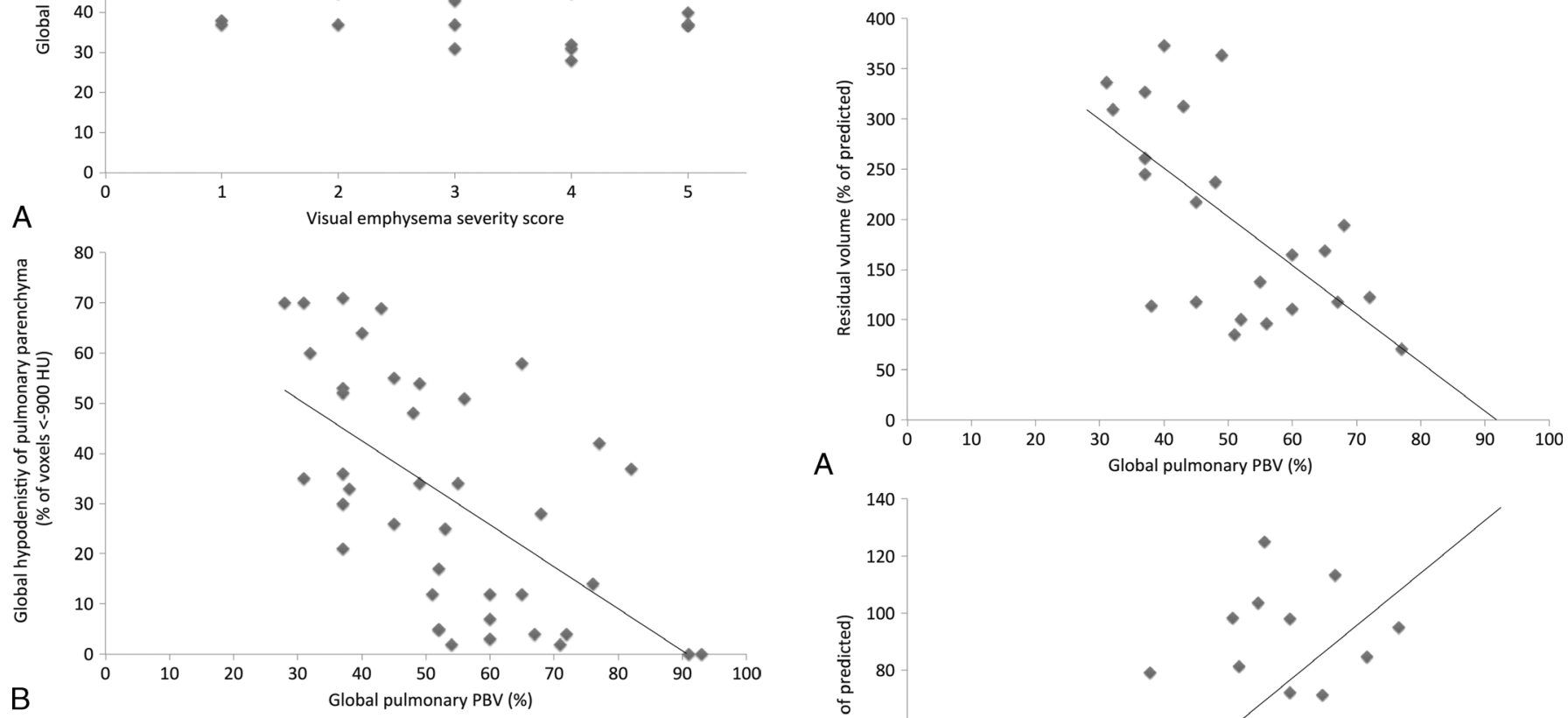

A
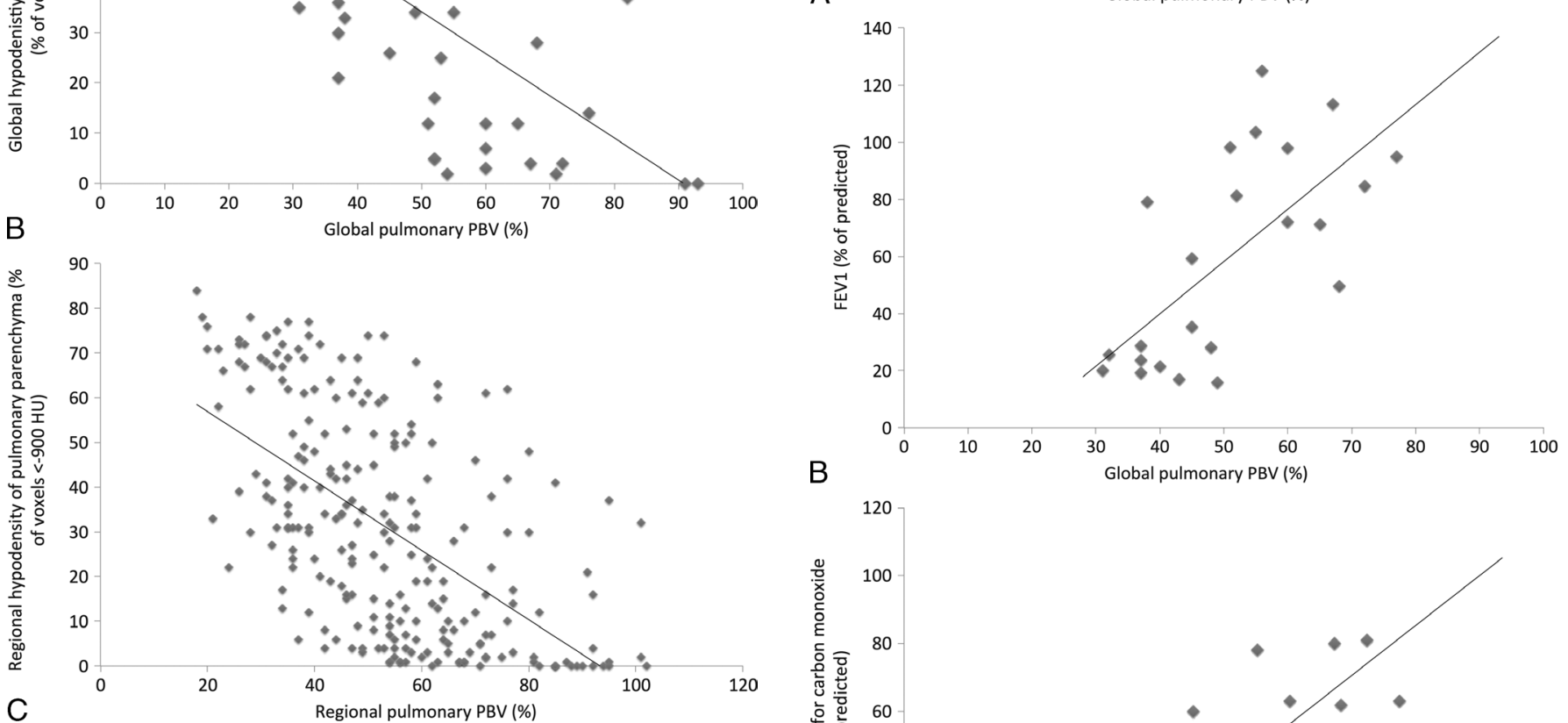

FIGURE 1. Correlation of the pulmonary PBV values with emphysema severity and parenchymal density. A, For all patients $(n=40)$, the global pulmonary PBV values are shown in relation to the visually determined severity of emphysema (1, minimal; 2 , mild; 3, moderate; 4 , severe; and 5, very severe). B, The global pulmonary PBV values are plotted against the overall percentage of voxels within the pulmonary parenchyma with a CT density less than $-900 \mathrm{HU}$ in the virtual noncontrast images in a per-patient analysis $(n=40)$. C, For a per-region analysis, the regional pulmonary PBV values are plotted against the percentage of voxels with a CT density less than $-900 \mathrm{HU}$ within that region. For each patient, the left and right lungs and the upper, middle, and lower regions were automatically defined by the software and were identical for density and PBV analyses $(n=240)$. The trend lines are shown, as determined by linear regression analysis.

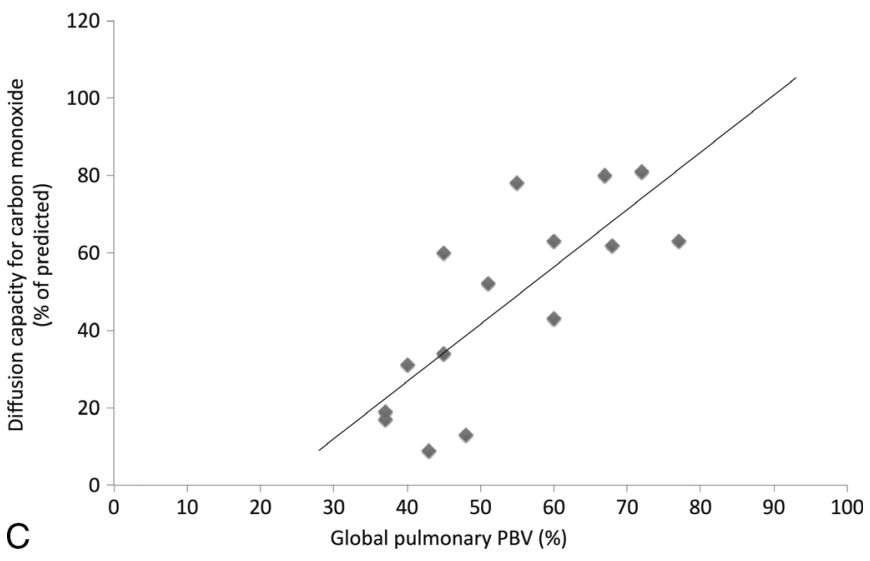

FIGURE 2. Correlation of the global pulmonary PBV values with PFTs. For each patient with PFTs performed $(n=23)$, the global pulmonary PBV values are plotted against RV in percentage of the predicted RV (A) and against $F E V_{1}$ in percentage of the predicted $\mathrm{FEV}_{1}(\mathrm{~B})$. The DLCO was only assessed in 15 patients. For these patients, the DLCO in percentage of the predicted DLCO in relation to the global pulmonary PBV (C) is shown. The trend lines are shown, as determined by the linear regression analysis. 

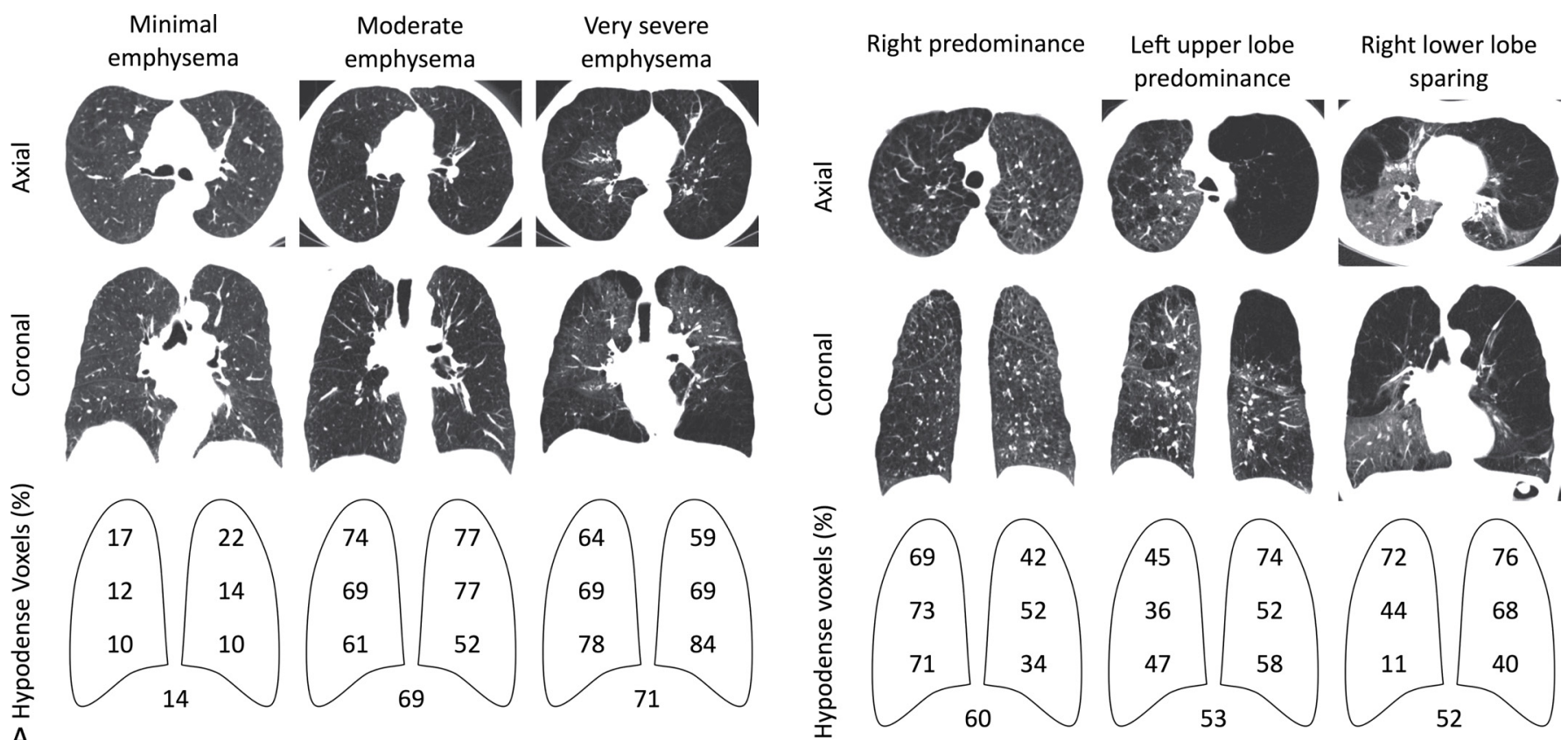

A
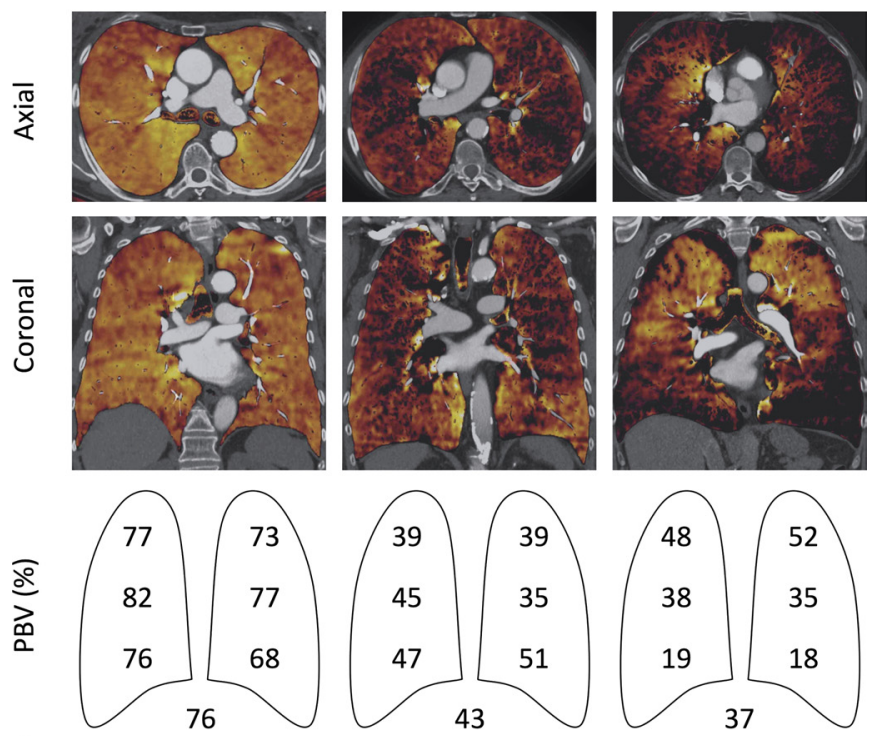

FIGURE 3. Representative images of and results from patients with pulmonary emphysema of varying severity. Representative images of and results from patients with visual emphysema severity scores of 1 (minimal), 3 (moderate), and 5 (very severe) are shown. A, Axial and coronal images similar to the standard $120-k V(p)$ CTPA images were generated with equal contributions from the $\operatorname{Sn} 140-k V(p)$ and the $100-k V(p)$ data set. Lung isolation, partitioning, and densitometric analysis were performed automatically. The percentage of voxels with a CT density less than $-900 \mathrm{HU}$ is shown for the upper, middle, and lower region of each lung and the entire lung parenchyma (below). B,

Color-coded iodine distribution maps are superimposed onto the CTPA images in axial and coronal orientations. After the manual definition of the pulmonary trunk as the reference vessel, lung isolation, partitioning, and enhancement analysis were performed automatically. Relative pulmonary parenchymal enhancement is shown for the entire pulmonary parenchyma and individual lung zones.
A
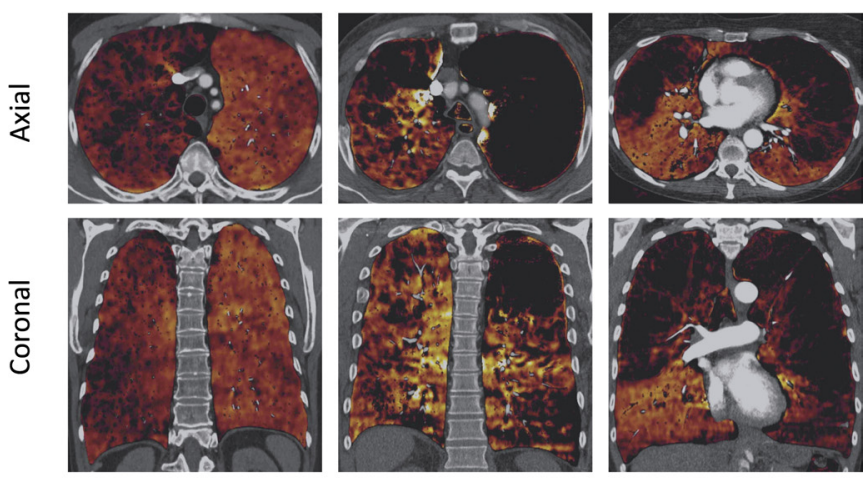

30
2
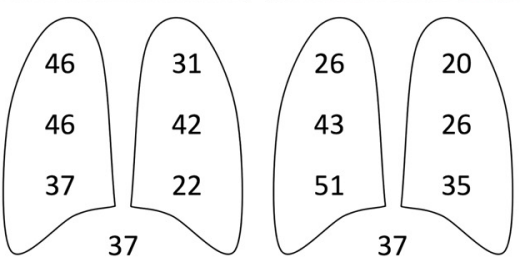

FIGURE 4. Regional heterogeneity of parenchymal changes and pulmonary PBV. Representative images of and results from the patients with marked regional differences in emphysema severity. A, Axial and coronal images similar to the standard $120-k V(p)$ CTPA images were generated with equal contributions from the $\mathrm{Sn} 140-\mathrm{kV}(\mathrm{p})$ and the $100-\mathrm{kV}(\mathrm{p})$ data set. Lung isolation, partitioning, and densitometric analysis were performed automatically. The percentage of voxels with a CT density less than $-900 \mathrm{HU}$ is shown for the upper, middle, and lower region of each lung and the entire lung parenchyma (below). B, Color-coded iodine distribution maps are superimposed onto the CTPA images in axial and coronal orientations. After the manual definition of the pulmonary trunk as the reference vessel, lung isolation, partitioning, and enhancement analysis were performed automatically. Relative pulmonary parenchymal enhancement is shown for the entire pulmonary parenchyma and individual lung zones. 
$P<0.001$; Fig. 2B). Again, this correlation was confirmed in the multivariate regression analysis $(\beta=0.40, P=0.006)$. The global PBV values strongly correlated with $\operatorname{DLCO}(r=0.80 ; P<0.001 ; n=15$; Fig. 2C). This correlation was stronger than the correlations between DLCO and the visual degree of emphysema $(r=0.70 ; P=0.004)$ and between DLCO and the percentage of voxels within the pulmonary parenchyma with a CT density less than $-900 \mathrm{HU}(r=0.70 ; P=0.003)$.

Representative images and PBV values of patients with pulmonary emphysema of varying severity are shown in Figure 3. Figure 4 shows images and PBV values of patients with pulmonary emphysema of marked regional heterogeneity.

\section{DISCUSSION}

Other imaging modalities such as perfusion magnetic resonance imaging, ${ }^{27}$ perfusion scintigraphy, ${ }^{10}$ and perfusion SPECT $^{28}$ can be used to quantify pulmonary perfusion. In COPD, changes in pulmonary perfusion assessed by perfusion scintigraphy or SPECT have been shown to correlate with the extent of emphysema as assessed by high-resolution CT and PFTs. ${ }^{10,28}$ However, these modalities are inferior to multidetector $\mathrm{CT}$ in the depiction of lung morphology. Coregistered SPECT/CT can combine detailed morphological assessment and perfusion analysis ${ }^{9}$ at the expense of a higher radiation dose. ${ }^{29}$ Dual-energy computed tomographic pulmonary angiography is dose neutral compared with conventional chest $\mathrm{CT}^{30}$ and combines excellent morphological depiction of lung parenchyma with pulmonary PBV maps, which correspond well with pulmonary perfusion on scintigraphy ${ }^{16}$ and SPECT ${ }^{17}$ images.

Interestingly, a number of studies using scintigraphy or SPECT have shown imperfect correlations between areas with most pronounced morphological changes and areas of decreased lung perfusion in pulmonary emphysema. ${ }^{5,10,31,32}$ Our data are in line with these previous results because we found only moderate correlations between the pulmonary PBV values and the visual and densitometric degree of parenchymal changes both in a per-patient analysis and a per-region analysis.

We conclude that the assessments of parenchymal morphology and pulmonary PBV on DE-CTPA are complementary sets of information that should be considered independently. In our study, the pulmonary PBV showed a moderately strong correlation with parameters of airflow obstruction and air trapping. The correlation strength is similar to what has been found for perfusion SPECT. ${ }^{28}$ Interestingly, our study showed a strong correlation between the pulmonary PBV values with DLCO as a global marker of lung function, which was stronger than the correlation between DLCO and the visual or densitometric degree of parenchymal changes. This may indicate that some morphologically normal lung areas are hypoperfused, thus diminishing their contribution to gas exchange. However, this particular correlation is based on the relatively small number of 15 patients and needs to be confirmed in larger, prospective studies.

The assessment of pulmonary perfusion in emphysema has gained clinical significance because a recent analysis demonstrated that LVRS reduces mortality in patients with upper lobe-predominant emphysema only if there is low, rather than high, perfusion to the upper lung on scintigraphy. ${ }^{10}$ Dual-energy computed tomographic pulmonary angiography with automated quantification of pulmonary $\mathrm{PBV}$ as a single comprehensive examination could potentially replace the combination of CT and scintigraphy or SPECT in the preoperative assessment of candidates for LVRS. For this and other clinical applications, the automated quantification of pulmonary PBV, as demonstrated in this study, renders DE-CTPA more useful for clinical routine by providing a quick, quantitative, and reader-independent estimation of global and regional pulmonary perfusion.

The results of our study have to be seen in the context of the study design. To avoid confounding, we excluded patients with emphysema who have any cardiopulmonary comorbidities. However, a significant proportion of patients with emphysema undergoing DE-
CTPA for suspected pulmonary embolism at our institution (an academic tertiary care hospital) has major cardiopulmonary comorbidities such as primary or metastatic pulmonary malignancy, congestive heart failure, pneumonia, or pleural effusions. The influence of these comorbidities on DE-CTPA pulmonary PBV values in an unselected patient population needs to be addressed in further studies.

Furthermore, this study did not compare the pulmonary PBV values to an external standard of reference such as ventilation/perfusion scintigraphy and the number of patients in this study is limited. Particularly, because of incomplete data sets available for the retrospective analysis, the correlations of imaging with PFTs and DLCO are only based on 23 and 15 patients, respectively, and need to be confirmed in larger studies.

Because the patients in this study underwent the CTPA examination with a suspicion of pulmonary embolism, we cannot entirely rule out that hidden pulmonary embolism not detected on CTPA may have confounded the PBV values. However, the sensitivity of multidetector CTPA for pulmonary embolism falls in the range of $83 \%$ to $89 \%$ with 4 -slice and 16 -slice scanners ${ }^{33}$ and is generally assumed to be even higher with more advanced scanners and the use of dual-energy technology. Therefore, it is very unlikely that a substantial percentage of the patients in this study had pulmonary emboli despite negative DE-CTPA findings.

In contrast to combined ventilation/perfusion scintigraphy and SPECT, the standard DE-CTPA used in this study is unable to directly assess pulmonary ventilation. However, the feasibility of a combined ventilation and perfusion dual-energy CT using inhaled xenon has been demonstrated in patients who underwent mechanical ventilation. ${ }^{34}$ Initial studies have demonstrated that ventilation dualenergy CT using inhaled xenon ${ }^{35}$ or krypton ${ }^{36}$ can identify ventilation abnormalities in patients with emphysema. Further studies are needed to investigate the diagnostic value of combined ventilation and perfusion dual-energy CT in patients with pulmonary emphysema, particularly in the preoperative assessment of candidates for LVRS.

\section{CONCLUSIONS}

Dual-energy computed tomographic pulmonary angiography can be used for simultaneous assessment of parenchymal morphology, parenchymal density, and pulmonary PBV in patients with pulmonary emphysema. On the basis of DE-CTPA data sets, automated quantification of pulmonary PBV provides an immediate, reader-independent quantification of global and regional lung perfusion, which correlates with several lung function parameters. This can be integrated into the clinical decision making regarding the medical, surgical, or endoscopic treatment of pulmonary emphysema.

\section{REFERENCES}

1. Mathers CD, Lopez AD, Murray CJL. The burden of disease and mortality by condition: data, methods, and results for 2001. In: Lopez AD, Mathers CD, Ezzati M, et al, eds. Global Burden of Disease and Risk Factors. Washington, DC: World Bank; 2006.

2. Ezzati M, Lopez AD. Estimates of global mortality attributable to smoking in 2000. Lancet. 2003;362:847-852.

3. Minai OA, Benditt J, Martinez FJ. Natural history of emphysema. Proc Am Thorac Soc. 2008;5:468-474

4. Soriano JB, Zielinski J, Price D. Screening for and early detection of chronic obstructive pulmonary disease. Lancet. 2009;374:721-732.

5. Sandek K, Bratel T, Lagerstrand L, et al. Relationship between lung function, ventilation-perfusion inequality and extent of emphysema as assessed by highresolution computed tomography. Respir Med. 2002;96:934-943.

6. Lee CW, Seo JB, Lee Y, et al. A pilot trial on pulmonary emphysema quantification and perfusion mapping in a single-step using contrast-enhanced dualenergy computed tomography. Invest Radiol. 2012;47:92-97.

7. Sciurba FC, Ernst A, Herth FJ, et al. A randomized study of endobronchial valves for advanced emphysema. N Engl J Med. 2010;363:1233-1244.

8. Criner GJ, Mamary AJ. Lung volume reduction surgery and lung volume reduction in advanced emphysema: who and why? Semin Respir Crit Care Med. 2010;31:348-364. 
9. Takenaka D, Ohno Y, Koyama H, et al. Co-registered perfusion SPECT/CT: utility for prediction of improved postoperative outcome in lung volume reduction surgery candidates. Eur J Radiol. 2010;74:465-472.

10. Chandra D, Lipson DA, Hoffman EA, et al. Perfusion scintigraphy and patien selection for lung volume reduction surgery. Am J Respir Crit Care Med. 2010;182:937-946.

11. Wu AS, Pezzullo JA, Cronan JJ, et al. CT pulmonary angiography: quantification of pulmonary embolus as a predictor of patient outcome-initial experience. Radiology. 2004;230:831-835.

12. Thieme SF, Johnson TR, Lee C, et al. Dual-energy CT for the assessment of contrast material distribution in the pulmonary parenchyma. AJR Am J Roentgenol. 2009; 193:144-149.

13. Remy-Jardin M, Faivre JB, Pontana F, et al. Thoracic applications of dual energy. Radiol Clin North Am. 2010;48:193-205.

14. Nance JW Jr, Henzler T, Meyer M, et al. Optimization of contrast material delivery for dual-energy computed tomography pulmonary angiography in patients with suspected pulmonary embolism. Invest Radiol. 2012;47:78-84.

15. Runge VM. Advances in diagnostic radiology. Invest Radiol. 2010;45: 823-826.

16. Thieme SF, Becker CR, Hacker M, et al. Dual energy CT for the assessment of lung perfusion-correlation to scintigraphy. Eur J Radiol. 2008;68:369-374.

17. Thieme SF, Graute V, Nikolaou K, et al. Dual energy CT lung perfusion imaging - correlation with SPECT/CT. Eur J Radiol. 2012;81:360-365.

18. Pansini V, Remy-Jardin M, Faivre JB, et al. Assessment of lobar perfusion in smokers according to the presence and severity of emphysema: preliminary experience with dual-energy CT angiography. Eur Radiol. 2009;19: 2834-2843.

19. Ferda J, Ferdova E, Mirka H, et al. Pulmonary imaging using dual-energy CT, a role of the assessment of iodine and air distribution. Eur J Radiol. 2011; 77:287-293

20. Sueyoshi E, Tsutsui S, Hayashida T, et al. Quantification of lung perfusion blood volume (lung PBV) by dual-energy CT in patients with and without pulmonary embolism: preliminary results. Eur J Radiol. 2011;80:e505-e509.

21. Deak PD, Smal Y, Kalender WA. Multisection CT protocols: sex- and agespecific conversion factors used to determine effective dose from dose-length product. Radiology. 2010;257:158-166.

22. Nikolaou K, Thieme S, Sommer W, et al. Diagnosing pulmonary embolism: new computed tomography applications. J Thorac Imaging. 2010;25:151-160.
23. Johnson TR, Krauss B, Sedlmair M, et al. Material differentiation by dual energy CT: initial experience. Eur Radiol. 2007;17:1510-1517.

24. Gietema HA, Muller NL, Fauerbach PV, et al. Quantifying the extent of emphysema: factors associated with radiologists' estimations and quantitative indices of emphysema severity using the ECLIPSE cohort. Acad Radiol. 2011;18:661-671

25. Zach JA, Newell JD Jr, Schroeder J, et al. Quantitative computed tomography of the lungs and airways in healthy nonsmoking adults. Invest Radiol. 2012;47: 596-602.

26. StatPrimer (Version 6.4). Available at: http://www.sjsu.edu/faculty/gerstman/ StatPrimer/correlation.pdf. Accessed July 21, 2012.

27. Hopkins SR, Wielputz MO, Kauczor HU. Imaging lung perfusion. J Appl Physiol. 2012;113:328-339.

28. Jogi J, Ekberg M, Jonson B, et al. Ventilation/perfusion SPECT in chronic obstructive pulmonary disease: an evaluation by reference to symptoms, spirometric lung function and emphysema, as assessed with HRCT. Eur J Nucl Med Mol Imaging. 2011;38:1344-1352.

29. Hara N, Onoguchi M, Takenaka K, et al. Assessment of patient exposure to x-radiation from SPECT/CT scanners. J Nucl Med Technol. 2010;38:138-148.

30. Schenzle JC, Sommer WH, Neumaier K, et al. Dual energy CT of the chest: how about the dose? Invest Radiol. 2010;45:347-353.

31. Suga K, Kawakami Y, Iwanaga H, et al. Assessment of anatomic relation between pulmonary perfusion and morphology in pulmonary emphysema with breath-hold SPECT-CT fusion images. Ann Nucl Med. 2008;22:339-347.

32. Suga K, Okada M, Kunihiro M, et al. Impaired lung 123I-MIBG uptake on SPECT in pulmonary emphysema. J Nucl Med. 2011;52:1378-1384.

33. Henzler T, Schoenberg SO, Schoepf UJ, et al. Diagnosing acute pulmonary embolism: systematic review of evidence base and cost-effectiveness of imaging tests. $J$ Thorac Imaging. 2012;27:304-314.

34. Thieme SF, Hoegl S, Nikolaou K, et al. Pulmonary ventilation and perfusion imaging with dual-energy CT. Eur Radiol. 2010;20:2882-2889.

35. Park EA, Goo JM, Park SJ, et al. Chronic obstructive pulmonary disease: quantitative and visual ventilation pattern analysis at xenon ventilation CT performed by using a dual-energy technique. Radiology. 2010;256:985-997.

36. Hachulla AL, Pontana F, Wemeau-Stervinou L, et al. Krypton ventilation imaging using dual-energy $\mathrm{CT}$ in chronic obstructive pulmonary disease patients: initial experience. Radiology. 2012;263:253-259. 